\title{
PERFIL EPIDEMIOLÓGICO DE MULHERES COM CÂNCER DE COLO DE ÚTERO TRATADAS EM HOSPITAL TERCIÁRIO
}

\section{EPIDEMIOLOGY OF WOMEN WITH CERVICAL CANCER TREATED IN A TERTIARY HOSPITAL}

\section{PERFIL EPIDEMIOLÓGICO DE MUJERES CON CÁNCER DE CUELLO UTERINO TRATADAS EN HOSPITAL TERCIARIO}

Caroline Ribeiro Pereira Favaro ${ }^{1}$, Lais Corsino Durant ${ }^{2}$, Tatiane da Silva Vaz Paterra ${ }^{3}$, Marislei Sanches Panobianco ${ }^{4}$, Thais de Oliveira Gozzo 5 .

\section{RESUMO}

Objetivo: caracterizar o perfil epidemiológico das mulheres com câncer de colo uterino atendidas em um hospital de referência em oncologia. Método: Analisaram-se 906 casos de câncer de colo de útero, atendidos no período de 2000 a 2013. Foram utilizados os testes Qui-quadrado e Kruskal-Wallis, para a comparação das variáveis; para a análise dos tempos, foi utilizado o teste de Mann Whitney e, para sobrevida, foram construídas curvas de Kaplan-Meier e comparadas via teste Log- Rank. Resultados: Das 906 mulheres analisadas, $68,6 \%$ possuíam ensino fundamental; $39,6 \%$ foram diagnosticadas em estádio clínico 0 . 0 cruzamento entre escolaridade e estadiamento apontou que, no estádio $034,8 \%$, possuíam ensino fundamental ou médio. A sobrevida global em cinco anos foi $56,5 \%$ e os casos diagnosticados em estádios avançados foram responsáveis pelo maior número de óbitos. Conclusão: A escolaridade influenciou no diagnóstico precoce, e o diagnóstico, em estádio avançado, ocasionou maior número de mortes.

Descritores: Neoplasias do Colo do útero; Perfil de Saúde; Enfermagem Oncológica; Sobrevivência; Prognóstico.

\section{ABSTRACT}

Objective: to characterize the epidemiological profile of women with cervical cancer treated at a referral hospital for oncology. Method: 906 cases of cervical cancer attended between 2000 and 2013 were used. Chi-square and Kruskal-Wallis tests were used to compare the variables; the Mann - Whitney test was used for the analysis of the times and Kaplan - Meier curves were constructed for survival and compared using the Log - Rank test. Results: Of the 906 women analyzed, 68.6\% had primary education; and $39.6 \%$ were diagnosed in clinical stage 0 . The cross-study between schooling and staging showed that, in stage 0 and in $39.6 \%$ of the cases, $34.8 \%$ had elementary or middle school education. Overall 5 -year survival was $56.5 \%$ and cases diagnosed in advanced stages were responsible for the highest number of deaths. Conclusion: The schooling influenced the early diagnosis and the diagnosis at an advanced stage caused a greater number of deaths.

Descriptors: Uterine Cervical Neoplasms; Health Profile; Oncology; Survivorship; Prognosis.

\section{RESUMEN}

Objetivo: caracterizar el perfil epidemiológico de las mujeres con cáncer de cuello uterino atendidas en un hospital de referencia en oncología. Método: Se analizaron 906 casos de cáncer de cuello uterino, atendidos en el período de 2000 a 2013 . Se utilizaron las pruebas Qui-cuadrado y Kruskal-Wallis para la comparación de las variables; para el análisis de los tiempos se utilizó la prueba de Mann-Whitney y para la supervivencia fueron construidas curvas de Kaplan-Meier y comparadas vía test Log-Rank. Resultados: De las 906 mujeres analizadas, el 68,6\% poseía educación básica; El 39,6\% fue diagnosticado en estadio clínico 0 . El cruce entre escolaridad y estadificación apuntó que, en el estadio 0 , el 39,6\% de los casos, el 34,8\% poseía educación básica o media. La supervivencia global en 5 años fue 56,5\% y los casos diagnosticados en estadios avanzados fueron responsables del mayor número de muertes. Conclusión: La escolaridad influenció en el diagnóstico precoz y el diagnóstico en estadio avanzado ocasionó mayor número de muertes.

Descriptores: Neoplasias del Cuello Uterino; Perfil de Salud; Enfermería Oncológica; Supervivencia; Pronóstico.

${ }^{1}$ Enfermeira, Mestre em Ciências pelo Programa de Pós-graduação Enfermagem em Saúde Pública da Escola de Enfermagem de Ribeirão Preto da Universidade de São Paulo. ${ }^{2}$ Enfermeira, Mestre em Ciências pelo Programa de Pós-graduação Enfermagem em Saúde Pública da Escola de Enfermagem de Ribeirão Preto da Universidade de São Paulo. ${ }^{3}$ Enfermeira, Mestre em Ciências pelo Programa de Pós-graduação Enfermagem em Saúde Pública da Escola de Enfermagem de Ribeirão Preto da Universidade de São Paulo. ${ }^{4}$ Enfermeira, Professor Associado do Departamento Materno Infantil e Saúde Pública da Escola de Enfermagem de Ribeirão Preto da Universidade de São Paulo. ${ }^{5}$ Enfermeira, Professor Associado do Departamento Materno Infantil e Saúde Pública da Escola de Enfermagem de Ribeirão Preto da Universidade de São Paulo. 


\section{INTRODUÇÃO}

O câncer de colo de útero é considerado um problema de saúde pública mundial, por exercer papel importante na morbimortalidade das mulheres, sendo um desafio às políticas públicas de países em desenvolvimento ${ }^{(1)}$.

Estatísticas apontam a ocorrência de 530 mil casos novos e $\mathbf{2 7 0}$ mil mortes por ano, no mundo, em decorrência do câncer de colo de útero $^{(2)}$ e estima-se que, em 2030, essa neoplasia seja responsável pela morte de 474 mil mulheres, sendo que $95 \%$ ocorrerão em países de média e baixa renda ${ }^{(1)}$. Na América Latina, a incidência do câncer de colo de útero é considerada uma das maiores do mundo, correspondendo a até $25 \%$ de todos os tipos de câncer em mulheres ${ }^{(4)}$.

No Brasil, o câncer de colo de útero é o terceiro tumor mais frequente na população feminina e estão previstos, para 2018-2019, 16.370 casos novos. De acordo com o Sistema de Informação sobre Mortalidade (SIM), o número de mortes pelo câncer de colo de útero, em 2013, foi de 5.430 mulheres $^{(5)}$.

O câncer de colo de útero, diagnosticado em estádio inicial, tem bom prognóstico. Entretanto países de média e baixa renda apresentam variação geográfica na incidência e na mortalidade por essa neoplasia. Essas diferenças podem ser atribuídas a níveis socioeconômicos, baixo acesso a exames de rastreamento e a programas de prevenção, condições de saúde precárias, entre outros ${ }^{(6)}$.

Aproximadamente $85 \%$ dos casos de CCU acontecem, nos países menos desenvolvidos da América Latina e, sobretudo, naqueles das regiões mais pobres da África, que apresentam taxas elevadas de incidência, enquanto os Estados Unidos, o Canadá, a Austrália, o Japão e países europeus apresentam as menores taxas. A mortalidade, por causa desse câncer, varia em até 18 vezes entre as diferentes regiões do mundo, com taxas de menos de dois por 100.000 mulheres, na Ásia Ocidental e de 27,6 na África oriental $^{(3)}$.

Identificar as características comuns e a sobrevida das mulheres tratadas com câncer de colo de útero, em nossos serviços, irá auxiliar no planejamento e controle dessa neoplasia em nossa população. Este estudo teve como objetivo caracterizar o perfil epidemiológico das mulheres com câncer de colo uterino atendidas em um hospital de referência em oncologia.

\section{METODOLOGIA}

Trata-se de um estudo transversal, realizado em um único hospital, com a utilização de dados de fonte secundária. O referido hospital é uma instituição de nível terciário, que se caracteriza como de grande porte, de alta complexidade, sendo referência para a região Nordeste do Estado de São Paulo, composta por quatro milhões de habitantes. Os usuários do Sistema Único de Saúde (SUS) representam 97\% dos atendimentos.

Para a coleta dos dados, foi utilizado o banco de dados do Registro Hospitalar de Câncer (RHC), que fornece informações, para o Registro de Câncer de Base Populacional (RCBP), que constitui um importante instrumento de apoio à formulação da Política Nacional de Atenção Oncológica, ao planejamento de saúde, à avaliação da qualidade da atividade assistencial e como recurso para a elaboração de pesquisas clinicas e trabalhos científicos ${ }^{(7)}$. Todos os dados que constam no RHC são provenientes dos prontuários de mulheres que receberam o diagnóstico de câncer de colo de útero.

Mulheres diagnosticadas com câncer de colo de útero, no período de janeiro de 2000 a dezembro de 2013, foram consideradas para este estudo. Os casos de câncer de colo de útero foram identificados, no banco de dados, por meio do CID-10 (Classificação Internacional de Doenças), sendo o C53 (C53.0-C53.1, C53.8C53.9) referente à essa neoplasia. Foram identificadas 909 mulheres diagnosticadas com o câncer de colo de útero, no período estudado, e três foram excluídas, pois apresentavam idade inferior a 18 anos, sendo o número final de casos analisados de 906 mulheres.

Este estudo foi organizado, de acordo com o Sistema TMN, para a classificação de tumores malignos e o tratamento foi categorizado em cirurgia, radioterapia, quimioterapia e hormonioterapia. Os casos foram avaliados, de acordo com a média de idade e grupos etários, estágio no diagnóstico e tratamentos recebidos.

A sobrevida global, em cinco anos, foi estimada, com base na data do primeiro diagnóstico (foi utilizada a data do resultado do exame anatomo-patológico). Para a última data de seguimento da mulher, foram consideradas: data do óbito registrada no banco de dados ou no prontuário hospitalar da mulher ou ainda de seu último atendimento no serviço.

A análise dos dados foi realizada, por meio do programa Statistical Package for the Social 
Sciences (SPSS 23.0) e incluiu análise descritiva e análise de sobrevida. Para a análise descritiva, foram utilizadas médias e desvios-padrões, para as variáveis quantitativas, e frequências absoluta e percentual, para as variáveis categóricas. Para a comparação de variáveis categóricas, em dois ou mais grupos, foi utilizado o teste Qui-quadrado. Para a comparação das médias de idade, foi utilizado o teste Kruskal-Wallis, que comparou as médias entre todos os estadiamentos. Para a análise dos tempos de espera, diagnóstico e tratamento, foi utilizado o teste de Mann Whitney. Para a análise de sobrevida, foram construídas as curvas de Kaplan-Meier e comparadas via teste Log- Rank. O teste foi realizado a $95 \%$ intervalo de confiança (IC95\%), e $P<0,05$ foi considerado estatisticamente significante.

Este estudo foi aprovado pelo Comitê de Ética (CAAE 39451714.0.0000.5393) sob o parecer de número 1.266 .933 atendendo às diretrizes e normas regulamentadoras de pesquisas envolvendo seres humanos, regulamentadas pela Resolução CNS 466/2012.

\section{RESULTADOS E DISCUSSÃO}

Dos 906 casos analisados, os resultados apontam que a faixa etária variou de 18 a 95 anos, e a predominante foi de 31 a 60 anos, correspondendo a 534 (58,9\%) mulheres, e a média foi de 48,8 anos ( $D P=16,4$ anos). Foi analisada a faixa etária, fora da recomendação do MS, a qual demonstrou que $47(5,2 \%)$ da população do estudo com diagnóstico do câncer de colo de útero estava na faixa de 18 a 24 anos e $170(18,8 \%)$ foram diagnosticadas acima dos 65 anos, somando um total de 217 (24\%) de mulheres diagnosticadas, fora do período recomendado pelo programa.

Das mulheres analisadas, $374 \quad(41,3 \%)$ possuíam ensino fundamental completo (Tabela 1).

Tabela 1 - Distribuição dos casos de câncer de colo de útero, segundo idade e escolaridade. Ribeirão Preto, SP, Brasil, 2015.

\begin{tabular}{lll}
\hline \hline Variáveis & $\mathbf{N}$ & \% \\
\hline Idade & 47 & 5,2 \\
$\leq 24$ & 98 & 10,8 \\
De 25 a 30 & 177 & 19,5 \\
De 31 a 40 & 174 & 19,2 \\
De 41 a 50 & 183 & 20,2 \\
De 51 a 60 & 57 & 6,3 \\
De 61 a 64 & 170 & 18,8 \\
$\geq 65$ & & \\
Escolaridade & & 10,8 \\
Analfabeto & 98 & 41,3 \\
Ensino Fundamental Completo & 374 & 27,0 \\
Ensino Fundamental Incompleto & 245 & 15,5 \\
Ensino Médio & 140 & 3,1 \\
Superior & 28 & 2,3 \\
Ignorado & 21 & \\
\hline \hline
\end{tabular}

Fonte: RHC.

Média de idade semelhante à observada foi encontrada, em estudo que traçou o perfil epidemiológico de 77.317 mulheres com câncer de colo de útero, no Brasil de 2000 a 2009. Obteve-se a média de 49,2 anos e $55,3 \%$ delas encontravam-se abaixo dos 50 anos de idade no momento do diagnóstico ${ }^{(8)}$. Semelhante também aos dados encontrados, em estudo realizado em Vitória-Espírito Santo, que analisou mulheres com estadiamento inicial, no período de 2000 a 2005, identificando a predominância da faixa etária entre 40 a 59 anos $(49,3 \%)^{(9)}$.

Vale destacar que o câncer de colo de útero é raro, em mulheres até 30 anos, e sua 
incidência eleva-se, progressivamente, até ter seu pico na faixa de 45 a 50 anos $^{(8)}$, o que aponta o aumento da idade, como um importante fator de risco para essa neoplasia ${ }^{(10)}$.

O estádio clínico mais observado foi o estádio 0 , diagnosticado em 359 (39,6\%) mulheres, mas, mesmo assim, destaca-se que 225 $(24,8 \%)$ delas foram diagnosticadas em estádios avançados (III e IV). O tipo celular mais incidente, nas mulheres analisadas, foi o Carcinoma Escamocelular Sem Outra Especificação (SOE), (Tabela 2).

Entre as modalidades de tratamento, a mais observada foi a cirurgia com 635 (70,1\%), e a mesma mulher pode ter sido submetida a mais de um tratamento no decorrer do seguimento no serviço (Tabela 2).

Tabela 2 - Distribuição dos casos de câncer de colo de útero, segundo estádio clínico, tipo celular, CID e tratamento. Ribeirão Preto, SP, Brasil, 2015.

\begin{tabular}{|c|c|c|}
\hline Variáveis & $\mathbf{N}$ & $\%$ \\
\hline \multicolumn{3}{|l|}{ Estádio clínico agrupado } \\
\hline 0 & 359 & 39,6 \\
\hline 1 & 137 & 15,1 \\
\hline II & 169 & 18,7 \\
\hline III & 145 & 16,0 \\
\hline IV & 80 & 8,8 \\
\hline $\mathrm{x}$ & 5 & 0,6 \\
\hline Y & 11 & 1,2 \\
\hline \multicolumn{3}{|l|}{ Tipo celular } \\
\hline Adenocarcinoma & 28 & 3,1 \\
\hline Adenocarcinoma, SOE* & 50 & 5,5 \\
\hline Carcinoma Escamocelular in situ, SOE & 56 & 6,2 \\
\hline Carcinoma & 93 & 10,3 \\
\hline Carcinoma in situ, SOE & 80 & 8,8 \\
\hline Carcinoma Escamocelular, SOE & 370 & 40,8 \\
\hline Neoplasia intraepitelial escamosa grau III & 218 & 24,1 \\
\hline Outros & 11 & 1,21 \\
\hline \multicolumn{3}{|l|}{ Tratamentos $^{+}$} \\
\hline Nenhum & 35 & 3,9 \\
\hline Cirurgia & 635 & 70,1 \\
\hline Radioterapia & 347 & 38,3 \\
\hline Quimioterapia & 325 & 35,9 \\
\hline Hormonioterapia & 13 & 1,4 \\
\hline \multicolumn{3}{|l|}{ CID de metástases ${ }^{\S}$} \\
\hline C67 (neoplasia maligna de bexiga) & 17 & 20,5 \\
\hline C34 (neoplasia maligna dos brônquios e pulmões) & 13 & 15,7 \\
\hline C22 (neoplasia maligna do fígado e vias biliares) & 11 & 13,3 \\
\hline C48 (neoplasia maligna dos tecidos moles do retroperitônio e peritônio) & 6 & 7,2 \\
\hline C41(neoplasia maligna dos ossos e cartilagens articulares) & 5 & 6,0 \\
\hline Outros $^{\ddagger}$ & 31 & 37,4 \\
\hline
\end{tabular}

Fonte: RHC. *SOE: Sem outra especificação. ${ }^{\dagger}$ A mesma mulher pode ter realizado mais de um tratamento. ${ }^{\ddagger}$ Outros sítios de metástases: ovário, vagina, vulva, pelve renal, cólon, reto, rim, encéfalo, medula, tireoide, gânglios linfáticos. ${ }^{\S} \mathrm{A}$ mesma mulher pode ter apresentado mais de um sítio de metástase. 
Observou-se que 46 (5\%) mulheres apresentaram metástase, sendo que, dentre elas, $28(60,9 \%)$ apresentaram pelo menos um sítio. Os principais sítios foram: bexiga 17 (20,5\%); pulmões 13 (15,7\%); fígado 11 (13,3\%); retroperitônio e peritônio seis $(7,2 \%)$ e ossos cinco (6\%). Segundo as normas da Fundação Oncocentro de São Paulo (FOSP), os sítios de órgãos vizinhos, acometidos pelo câncer, não são classificados como invasão tumoral, mas sim como metástases $^{(7)}$.

Um estudo que utilizou os dados do Registro de Câncer de Base Populacional (RCBP) de quatro capitais brasileiras, no período de 1990 a 2004, mostrou uma tendência, para o decréscimo no câncer invasor, e o crescimento do carcinoma in situ entre grupos de mulheres estudadas que podem indicar resultados positivos das ações de rastreamento, para o câncer de colo de útero, nas cidades de Fortaleza, Porto Alegre e São Paulo ${ }^{(11)}$. No presente estudo, o carcinoma in situ (estádio 0) e o estádio I foram diagnosticados em $54,7 \%$ das mulheres, dado semelhante ao que se refere a esse tipo de lesão relatado pelo INCA, em que se afirma que, atualmente, $44 \%$ dos casos diagnosticados são de câncer in situ ${ }^{(5)}$.

O estádio clinico 0 , o mais frequente entre as mulheres analisadas, pode demonstrar que a rede de saúde que assiste os municípios, aos quais o serviço em estudo é referência, tem implementado ações adequadas em relação ao rastreamento. No entanto necessitam revisar suas condutas e melhorar a abordagem dessas mulheres, pois o diagnóstico, em fase avançada, infelizmente, ainda persiste e apresenta alto índice de mortalidade por câncer de colo de útero em mulheres.

Tendo em vista que das 906 pacientes 280 morreram, em decorrência do câncer, ou seja, $30,9 \%$ da população do estudo, $24,8 \%$ estão relacionadas a estádios avançados, o que evidenciou a alta mortalidade associada a esses estádios. Representam dados que sugerem rastreamento oportunístico, que se caracteriza pela realização do exame, quando a mulher procura o serviço de saúde e, além disso, o diagnóstico tardio, também, sugere insuficiência na qualidade dos serviços oferecidos ${ }^{(12)}$, o que poderia ser minimizado se, no país, houvesse programa de rastreamento organizado para o câncer de colo de útero.

A análise dos casos, segundo o estádio clínico e a escolaridade, demonstrou que, no estadiamento 0, encontravam-se $359(39,6 \%)$ das mulheres diagnosticadas com câncer de colo de útero, sendo que $41 \%$ apresentavam ensino fundamental completo (Tabela 3).

Tabela 3 - Distribuição dos casos de câncer de colo de útero, segundo estadiamento clínico e escolaridade. Ribeirão Preto, SP, Brasil, 2015.

\begin{tabular}{|c|c|c|c|c|c|c|}
\hline EC/Escolaridade & Analfabeto & $\begin{array}{c}\text { Ensino } \\
\text { Fundamental } \\
\text { Incompleto }\end{array}$ & $\begin{array}{c}\text { Ensino } \\
\text { Fundamental } \\
\text { Completo }\end{array}$ & $\begin{array}{l}\text { Ensino } \\
\text { Médio }\end{array}$ & Superior & Ignorado \\
\hline 0 & 21 & 103 & 144 & 68 & 15 & 8 \\
\hline I & 14 & 35 & 57 & 21 & 6 & 4 \\
\hline II & 26 & 42 & 66 & 31 & 2 & 2 \\
\hline III & 22 & 38 & 63 & 13 & 3 & 4 \\
\hline IV & 10 & 25 & 35 & 7 & 2 & 1 \\
\hline$x$ & 3 & 0 & 2 & 0 & 0 & 0 \\
\hline$Y$ & 2 & 2 & 7 & 0 & 0 & 0 \\
\hline
\end{tabular}

Fonte: RHC.

A relação entre estádios clínicos avançados e a escolaridade, também, foi observada em estudo realizado com objetivo de analisar os fatores determinantes do diagnóstico, em estádio avançado, em mulheres com câncer de colo de útero no Brasil; observou-se, como principal fator associado, o tipo histológico "carcinoma de células escamosas". Também foi verificado que as disparidades socioeconômicas presentes no país estão associadas ao estádio avançado da doença; mulheres com 50 anos de idade ou mais, vivendo sem companheiro, de raça/cor preta e com baixa escolaridade ${ }^{(9)}$. 
As pessoas com menor renda e escolaridade, por serem exatamente as mais expostas aos fatores de risco e com menor acesso às informações e aos serviços de saúde, são consideradas as mais vulneráveis às doenças crônicas não transmissíveis, dentre elas o câncer $^{(13)}$. É provável que essa associação seja uma realidade sociodemográfica brasileira, em especial, em mulheres que buscam atendimento no serviço público de saúde ${ }^{(14)}$.

Quando realizado o cruzamento entre o estádio clínico e a ocorrência de óbito, identificase que os estádios mais avançados, III e IV, foram responsáveis pelo maior número de morte entre as mulheres estudadas. Das 359 (39,6 \%) mulheres diagnosticadas em estádio 0, apenas 14 $(3,9 \%)$ foram a óbito. Já em relação aos estádios mais avançados, das 145 (16\%) mulheres em estádio III, 87 (60\%) foram a óbito e das 80 (8,8\%) mulheres diagnosticadas em estádio IV, 67 $(83,75 \%)$ faleceram.
De acordo com as análises estatísticas, observou-se que a média, em dias corridos entre a primeira consulta e o diagnóstico de câncer de colo de útero foi de 13,6 dias ( $D P=48,3$ ); entre o diagnóstico e o início do tratamento foi de 58,4 dias $(D P=76,7)$ e da primeira consulta até o último acompanhamento, seja por alta médica, óbito ou abandono do tratamento foi de 999,9 dias, sendo o mínimo de zero e o máximo 5538 dias.

Das 906 pacientes analisadas, 38 (4,2\%) chegaram ao serviço com diagnóstico do câncer e 868 (95,8\%) sem diagnóstico. Portanto, para a análise dessas variáveis, foi utilizado o $\mathrm{N}$ de 868 mulheres.

Para análise de sobrevida, foi realizado o teste de Log-Hank, o qual identificou que, em 60 meses (cinco anos), a probabilidade de desenvolver o evento é 56,5\% (Figura 1).

Figura 1 - Curva de Kaplan-Meier demostrando sobrevida geral em cinco anos de mulheres com câncer de colo de útero. Ribeirão Preto, SP, Brasil, 2015.

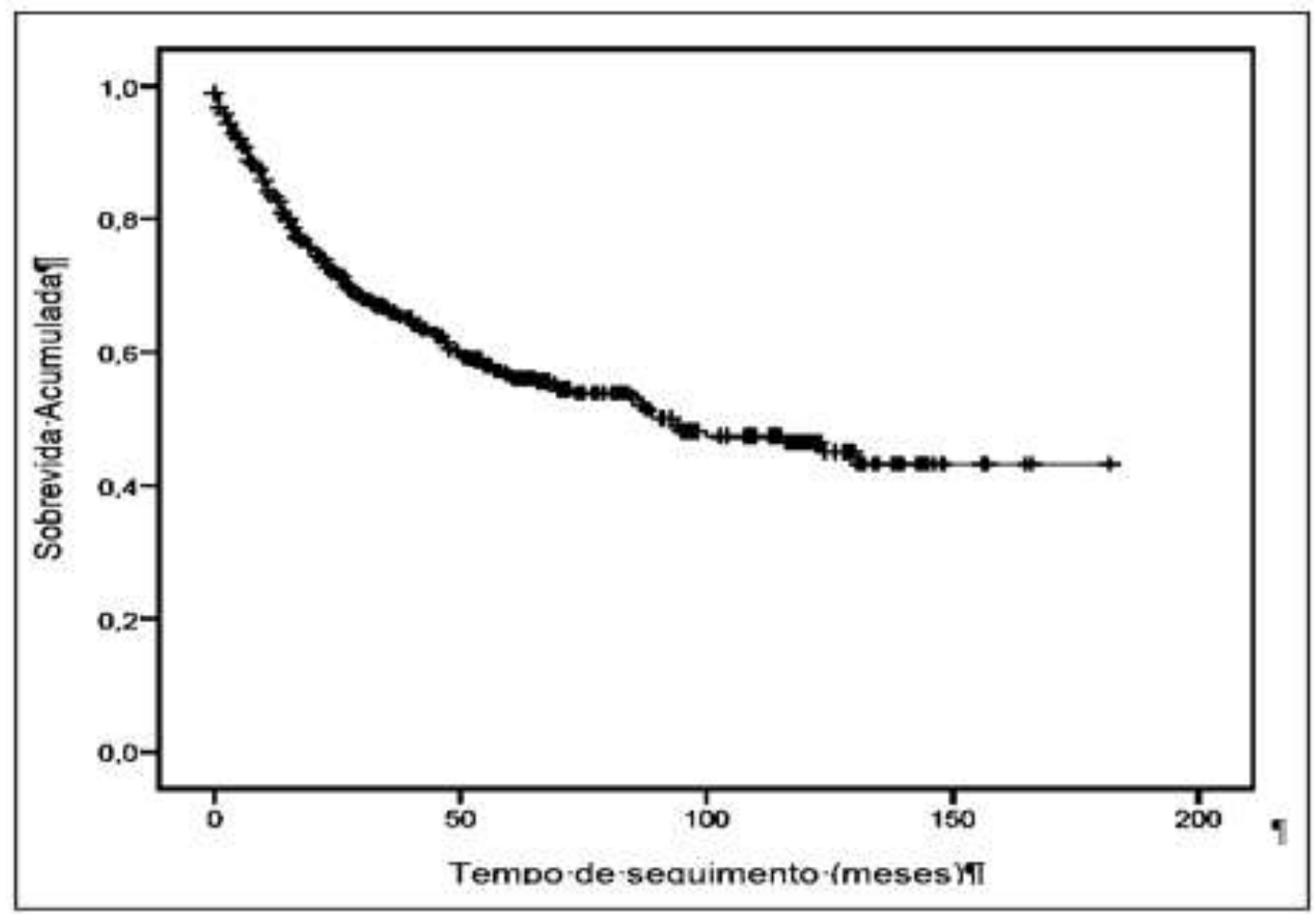

De acordo com estádio, a sobrevida foi de $96,1 \%$, para o estádio 0 e de $16,3 \%$, para o estádio IV, demostrando que quanto mais avançado o estádio, menor a sobrevida (Figura 2). 
Figura 2 - Curva de Kaplan-Meier demostrando sobrevida geral em cinco anos em relação ao estadiamento do câncer de colo de útero. Ribeirão Preto, SP, Brasil, 2015.

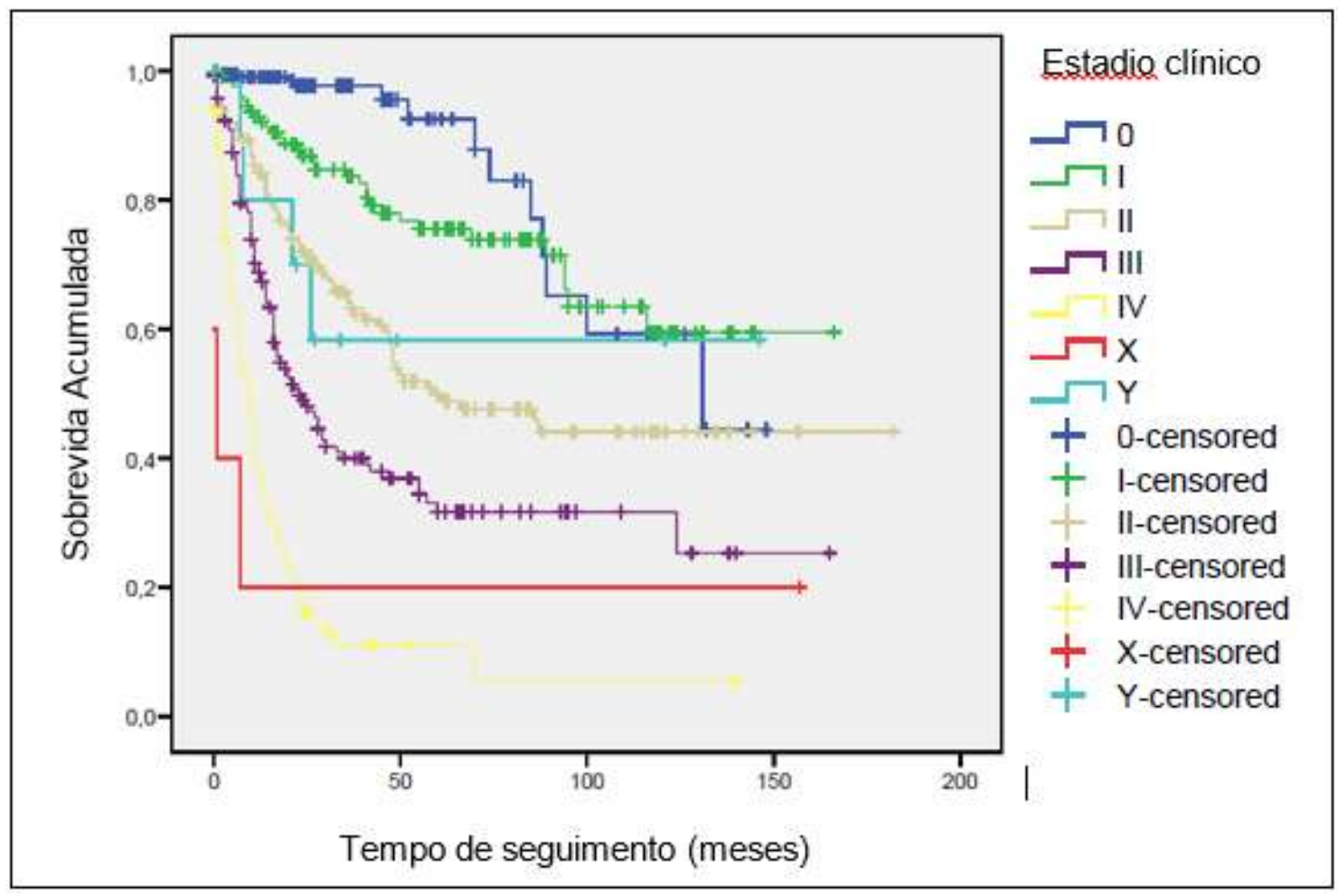

Ao realizar o Teste de Mann-Whitney, para a variável tempo de observação, obteve-se $p=$ 0,000 , demonstrando que essa variável foi estatisticamente significativa. No entanto, para as variáveis tempo de espera $(p=0,917)$ e tempo de diagnóstico/tratamento $(p=0,895)$, não foram estatisticamente significativas.

No contexto mundial, de forma geral, a sobrevida, em cinco anos para o câncer de colo de útero, apresentou melhora, ao longo dos anos, variando de menos de $50 \%$ para mais de 70\%. No Brasil, de 2005 a 2009, a sobrevida ficou em torno de $61 \%{ }^{(5)}$. No presente estudo, a sobrevida global, em cinco anos, foi de $56,5 \%$.

A média, em dias, entre o diagnóstico e o início do tratamento de câncer de colo de útero foi de 58,42 dias, com $D P=76,72$ dias. Esse valor está dentro da Lei dos 60 dias para o tratamento de câncer (Lei 12.732/12). A presente lei entrou em vigor, em 2012 e assegura aos pacientes com câncer o início do tratamento em, no máximo, 60 dias, após a inclusão da doença em seu prontuário, no SUS ${ }^{(15)}$. Visto que o valor variou entre 53,31 dias e 63,53 dias, com intervalo de confiança de $95 \%$, a presente lei não apresentou influência significante, para a população do estudo, pois os valores em dias já se enquadravam dentro do período imposto pela lei, mesmo antes de ela entrar em vigor.

Destaca-se que a implementação dos programas de prevenção tem sido a principal responsável pela modificação da taxa de incidência do câncer de colo de útero, na maioria dos países, em processo de transição socioeconômica ${ }^{(16)}$, em que o Brasil se encontra. No entanto, atingir o nível adequado de cobertura da população-alvo continua um desafio, para países em desenvolvimento, sendo o conhecimento dos fatores associados com a realização do Papanicolaou essencial à construção de estratégias, para a redução da incidência e a mortalidade por câncer de colo de útero ${ }^{(17)}$. Neste sentido, nota-se a necessidade de um olhar voltado à Atenção Primária à Saúde, focado nas ações de rastreamento, ampliação do acesso e direcionamento à população-alvo.

Um estudo que analisou informações relatadas por mulheres brasileiras entre 25 e 64 anos, provenientes da Pesquisa Nacional de Saúde (PNS), realizada em 2013, nas 26 capitais brasileiras e Distrito Federal e comparadas às informações do Sistema Vigitel (Sistema de Vigilância de Fatores de Risco, para Doenças Crônicas não Transmissíveis por Inquérito 
Telefônico), concluiu que a cobertura do exame de Papanicolau está abaixo de $80 \%{ }^{(18)}$.

0 êxito das ações de rastreamento depende de fatores como a mobilização e informação da população, alcance da meta de cobertura da população-alvo e, além disso, é necessário garantir o acesso ao diagnóstico e tratamento, monitorando e gerenciando as ações empregadas ${ }^{(19)}$. De acordo com as recomendações europeias, para alcançar esse êxito, é necessário obter uma cobertura da população-alvo de, pelo menos, $95 \%$ dessas mulheres, uma cobertura do exame de, pelo menos, $70 \%$ ( $85 \%$ é o desejável) e uma taxa de participação de, pelo menos $70 \% \quad(85 \%$ é o desejável) ${ }^{(20)}$.

Observou-se, neste estudo, que o perfil epidemiológico e a sobrevida das mulheres com câncer de colo de útero, inserido no RHC em estudo, condiz com o que está descrito na literatura. Entretanto estudo retrospectivo, realizado com banco de dados, apresenta limitações, como o viés de seleção, na inclusão de pacientes, para a análise e possíveis perdas de informações durante o acompanhamento.

\section{CONCLUSÃO}

O presente estudo mostrou que, mesmo com programas de prevenção e rastreamento precoce do câncer de colo de útero disponível na rede de saúde, o diagnóstico, em estádio avançado, persiste, diminuindo a sobrevida e causando a morte de centenas de mulheres. 0 grau de escolaridade revelou-se como o principal fator, para o acometimento por câncer de colo de útero, visto que, quanto maior o grau de informação e esclarecimento, menos expostas tornam-se as mulheres aos fatores de risco. 0 diagnóstico, em estádio inicial, proporciona maior sobrevida; consequentemente, menos mulheres morrem por essa neoplasia, o que foi observado neste estudo.

Diante disso, é evidente a necessidade de avaliação da qualidade e gestão do programa de rastreamento do câncer de colo de útero e as políticas públicas relacionadas à essa questão, pois fica evidente a dificuldade, para 0 diagnóstico precoce desta neoplasia, comprometendo a possibilidade de cura das mulheres afetadas.

Sugere-se que estudos futuros avaliem a adesão aos programas de rastreamento do câncer de colo de útero, a qualidade do cuidado prestado e da acessibilidade ao sistema de saúde, assim como avaliem os avanços diagnósticos e terapêuticos, além de comparações entre o serviço público e a saúde suplementar no atendimento a essas mulheres.

\section{AGRADECIMENTOS}

Registro Hospitalar de Câncer do Hospital das Clínicas da Faculdade de Medicina de Ribeirão Preto da Universidade de São Paulo.

\section{REFERÊNCIAS}

1- Bray F, Ferlay J, Soerjomataram I, Siegel RL, Torre LA, Jemal A. Global cancer statistics 2018: GLOBOCAN estimates of incidence and mortality worldwide for 36 cancers in 185 countries. CA Cancer J Clin 2018; 68(6):394-424. DOI: 10.3322/caac.21492

2- Wang SM, Qiao YL. Implementation of cervical cancer screening and prevention in China: Challenges and reality. Jpn J Clin Oncol. 2015;45(1):7-11. DOI: 10.1093/iico/hyu188

3- Brasil. Ministério da Saúde. Divisão de deteç̧ão precoce e apoio à organização de rede: Diretrizes brasileiras para o rastreamento do câncer do colo do útero. $2 a$ ed. rev. e atual. Rio de Janeiro: Inca; 2016.

4- Teixeira LA. Dos gabinetes de ginecologia às campanhas de rastreamento: a trajetória da prevenção ao câncer de colo do útero no Brasil. Hist Ciênc Saúde 2015;22(1):221-39. DOI: 10.1590/S0104-59702015000100013

5- Instituto Nacional de Câncer José Alencar Gomes da Silva (INCA). Estimativa 2018. Rio de Janeiro: INCA; 2018. Disponível em: http://www.inca.gov.br/estimativa/2018/sintesede-resultados-comentarios.asp

6- Brasil. Ministério da Saúde. Controle do câncer do colo do útero: Detecção precoce. Rio de Janeiro: INCA; 2018.

7- Fundação Oncocentro de São Paulo (FOSP). Registro hospitalar de câncer: Conceitos, rotinas e instruções de preenchimento. São Paulo: FOSP; 2013.

8- Thuler LCS, Bergmann A, Casado L. Perfil das pacientes com câncer do colo do útero no Brasil, 2000-2009: Estudo de base secundária. Rev Bras Cancerol 2012 [citado em 12 out 2018]; 58(3):351-7. Disponível em: http://www1.inca.gov.br/rbc/n 58/v03/pdf/04 a rtigo perfil pacientes cancer colo utero brasil 20002009 estudo base secundaria.pdf 9- Mascarello KC, Silva NF, Piske MT, Viana KCG, Zandonade E, Amorim MHA. Perfil sociodemográfico e clínico de mulheres com câncer 
do colo do útero associado ao estadiamento inicial. Rev Bras Cancerol 2012 [citado em 31 out 2018]; 58(3):417-26. Disponível em: http://www1.inca.gov.br/rbc/n 58/v03/pdf/11 a rtigo perfil sociodemografico clinico mulheres cancer colo utero associado estadiamento inic ial.pdf

10- Katari AC, Sharma JD, Krishnatreya $M$, Baishya N, Barmon D, Deka P, et al. A survival study of uterine cervical patients in the North East India: Hospital-cancer registry-based analysis. J Cancer Res Ther 2018;14(5): 1089-93. DOI: $10.4103 / 0973-1482$

11- Ayres ARG, Silva GA, Guimarães RM. Tendência da incidência de câncer do colo do útero invasor em quatro capitais brasileiras: Dados dos registros de câncer de base populacional, 1990-2004. Cad Saúde Colet. 2013; 21(3):289-95. DOI: $\quad 10.1590 /$ S1414$\underline{462 \times 2013000300009}$

12- Carvalho PG, O'Dwer G, Rodrigues NCP. Trajetórias assistenciais de mulheres entre diagnóstico e início de tratamento do câncer de coli uterino. Saúde Debate 2018;42(118):687701. DOI: $10.1590 / 0103-110420181181$

13- Ansari F. Different socioeconomic factors associated with cervical cancer. Int J Eng Appl Sci. 2016 [citado em 31 out 2018];3(1):36-8. Disponível em:

https://webcache.googleusercontent.com/search ?q=cache: VKGyP98bRgJ:https://www.ijeas.org/ download data/IJEAS0301021. pdf $+\& c d=4 \&$ hl=ptBR\&ct=clnk\&gl=br

14- Malta DC, Silva Júnior JB. O plano de ações estratégicas para o enfrentamento das doenças crônicas não transmissíveis no Brasil e a definição das metas globais para o enfrentamento dessas doenças até 2025: Uma revisão. Epidemiol Serv Saúde 2013;22(1):151-64. DOI: 10.5123/S167949742013000100016

15- Brasil. Lei no 12.732, de 22 de novembro de 2012. Dispõe sobre o primeiro tratamento de paciente com neoplasia maligna comprovada e estabelece prazo para seu início. Diário Oficial da União 2012. Disponível em: http://www.planalto.gov.br/ccivil 03/ ato20112014/2012/lei//12732.htm

16- Organização Mundial da Saúde (OMS). Folha Informativa - Câncer. Brasília: OMS; 2018.

17- Musa J, Achenbach C, O'Dwyer LC, Evans CT, McHugh M, Hou L, et al. Effect of cervical cancer education and provider recommendation for screening on screening rates: A systematic review and meta-analysis. PLoS One 2017;12(9):e0183924.

DOI:

10.1371/journal.pone.0183924

18- Oliveira MM, Andrade SSCA, Oliveira PPV, Silva GA, Silva MMA, Malta DC. Cobertura de exame Papanicolaou em mulheres de 25 a 64 anos, segundo a Pesquisa Nacional de Saúde e o Sistema de vigilância de fatores de risco e proteção para doenças crônicas por inquérito telefônico, 2013. Rev Bras Epidemiol. 2018; 21:111. DOI: $10.1590 / 1980-549720180014$

19- Pimple AS, Mishra GA. Global strategies for cervical cancer prevention and screening. Minerva Ginecol. 2019;71(4):313-20. DOI: 10.23736/S0026-4784.19.04397-1.

20- Derchain S, Teixeira JC, Zeferino LC. Organized, population-based cervical cancer screening program: It would be a good time for Brazil now. Rev Bras Ginecol Obstet. 2016;38(4):161-3. DOI: 10.1055/s-0036-1582399

Nota: Artigo resultante de pesquisa de dissertação.

Recebido em: 10/01/2019

Aprovado em: 13/09/2019

Endereço de correspondência:

Thais de Oliveira Gozzo

Av. Bandeirantes, 3900

Campus Universitário - Bairro Monte Alegre

CEP: 14040-902 - Ribeirão Preto/MG - Brasil

E-mail: thaisog@eerp.usp.br 